\title{
Issues in Periodontal Research in India
}

\author{
Agraja Patil ${ }^{1}$, Swapna Mahale ${ }^{2}$, Chaitanya Joshi ${ }^{3}$, Prutha Vaidya ${ }^{4}$ \\ ${ }^{I}$ (Post- Graduate Student, Department of Periodontology, MGV KBH Dental College, Nashik) \\ ${ }_{2}^{2}$ (Professor, Guide and HOD, Department of Periodontology, MGV KBH Dental College, Nashik) \\ ${ }^{3}$ (Post- Graduate Student, Department of Periodontology, MGV KBH Dental College, Nashik) \\ ${ }^{4}$ (Post-Graduate Student, Department of Periodontology, MGV KBH Dental College, Nashik)
}

\begin{abstract}
Periodontitis is a multi-factorial disease which has affected mankind since the dawn of an era. Prevalence of periodontal disease has considerably increased over a period of time. Periodontal research has progressed tremendously worldwide. In India in spite of increased periodontal research, many problems are faced by the clinicians. It leads to improper recording of the results and resultant failure to spread the knowledge that would help in understanding the subject better. This is a major setback in the current era of evidenced based clinical practice. Hence in this review, an attempt is made to discuss and emphasize these unsolved issues pertaining to epidemiology, disease activity and therapy.
\end{abstract}

Keywords: Epidemiology, Ethics, Finance, Pathogenesis, Solution, Therapy

\section{Introduction}

Use of Information technology for data acquisition, interprétation and dissemination of result has spurred increase in research activities worldwide, including India. Primary aim of any research paper is to improve the clinical management of periodontitis, which is a hugely prevalent and unpleasant inflammatory disease and it can have a significant impact on quality of life.

As defined by William J Gies, Research in its highest expression is an open minded inquiry for truth, to be found unreservedly for the instruction, information and welfare of all. ${ }^{[1]}$ Research is a quest for knowledge through diligent search (or) investigation (or) experimentation aimed at the discovery and interpretation of new knowledge. ${ }^{[2]}$ Triad of Evidence based dentistry includes individual clinical expertise, patients' values and expectations and best available evidence to be utilized for improved patient outcomes. Hence, there is a need for generation of evidence.

\section{Issues in Clinical research}

Clinicians, patients, policy makers and treatment funders all need to know which treatments are the most effective for managing particular clinical conditions. Randomized controlled trials (RCT) provide the highest level of scientific evidence regarding the efficacy of specific treatments. There is an intense requirement to achieve clinically relevant results than statistically significant.

Steps in an Randomised Controlled Trial include selection of patient population, randomisation, allotment of test and control groups, intervention, follow-up, analysis of collected data. ${ }^{[3]}$ Various issues that need to be addressed when undertaking these trials are regarding inclusion/ exclusion criteria, clauses and authorship amongst examiners in the consent form, randomization, need to care for well-being of all the patients involved, elimination of bias by blinding during data collection and its management.

\section{Research Facilities in India}

Oral diseases are widely prevalent in India. Though, not life-threatening, these diseases are often very painful, expensive to treat and cause loss of teeth. Periodontics as a specialty received very little attention during 1960-70s. Little was known about the pathogenesis and management of periodontal diseases. The scientific advances made since then have constructively helped us to understand the disease and alter our treatment accordingly.

Most of the research is carried out in dental institutions as a part of post graduate studies. There is nonavailability of sophisticated equipments due to a wide knowledge gap between the developed world and developing countries. No uniform modernization of laboratory facilities all over India. No skilled, trained, competent and willing technicians to operate sophisticated machines, hence they become difficult to maintain with no efficient use of those facilities. Spare parts and trained trouble-shooters need to be imported. Migration of skilled researchers and technicians to well developed countries in search of lucrative jobs is seen. Lack of motivation amongst clinicians \& academicians is disheartening. Meager encouragement for interested researchers in dental institutions is seen. This type of critical issues can be addressed by the following ways:

1. National policies which make the entire dental practice and research appealing 
2. Commercialization of various sectors

3. Simplifying the rules and regulations

4. Minimizing rejection of proposals

\section{Finance}

No adequate funding for research which hampers young researchers from conducting "dream projects". There is deficiency of technical knowledge of attaining funds among researchers which has led to disappointment and loss of desired results. The present cost of dental education as well as the rate of tuition expense increase is simply not sustainable. Innovation is desperately required to improve dental education, the quality of comprehensive oral health care, and access to quality care for everyone. Very less proportion of health funds is allotted to dental institutions by state governments out of which there is an improper handling of the limited amount. This financial problem has to be dealt by bridging the gap between researchers and finance organizations by creating awareness. Different funding agencies are available like

1. Indian Medical Council for Research,

2. International Clinical Dental Research organization (2008)

3. Multinational companies with research facilities in India

4. Ayurvedic and Pharmaceutical companies conducting test trials for their products

\section{a. Epidemiology/ prevalence}

\section{Issues in disease activity}

In 1950's-60's, indices used to measure periodontal disease were Periodontal Index of Russell (1967) ${ }^{[4]}$, and The Periodontal Disease Index of Ramfjord (1967) ${ }^{[5]}$. If periodontitis is left untreated, it progresses to eventual loss of tooth. In a National survey done in United States (1981), individuals of age 18-65 years were included. Prevalence examined in individuals $>45$ years. Prevalence is much lower than expected and disease was in small proportion with severe form. ${ }^{[6]}$

Studies in third world countries showed low plaque and calculus levels with little or no periodontitis. Chronically high levels of plaque and calculus showed higher levels of more severe periodontitis. However, studies by Baelum (1986) ${ }^{[7]}$ and Reddy et al. (1986) ${ }^{[8]}$ showed non- relevance of surface deposits to occurrence of disease. There are three basic critical issues that arise:

1. Is prevalence changing?

Decreasing prevalence would have enormous implications for dental education, dental practice, and public health. Severity is less than previously observed. But prevalence is still unclear. There is no universal data from US and other countries. Regular nation-wide surveys regarding prevalence can be conducted to solve this dilemma.

2. To determine the identity and characteristics of periodontitis patients

There are differences in race, ethnicities and nationalities. In previously done surveys there was no determination of prevalence in population sub-groups. No identification of highly susceptible groups. Study by Hughes et al. (1982) showed severe disease in blacks, low-socio economic groups and low education. More association studies are needed.

3. Validity of oral hygiene

Baelum (1986) in Tanzania and Pemba Islands in individuals not exposed to any oral hygiene methods. Severe plaque and calculus was found with no gross periodontal disease. Such studies show a complex relationship between $\mathrm{OH}$ and periodontal status. Only bacteria might not be responsible for disease, other factors need to be considered like Host-relationship (Offenbacher, 1980), Environment (Marsh, 1986), Genetics (Kornman, 2008). ${ }^{[6]}$

\section{b. Issues in Microbiology/Etiology}

In early 1960's, plaque and calculus were considered as etiologic factors but no specific mechanism was known. First classic experimental gingivitis studies were conducted by Loe and co-workers in $1965^{[9]}$. Beagle dog studies by Lindhe et al. (1975) demonstrated that periodontitis is caused by bacteria. It gave rise to Non-specific plaque hypothesis by Miller (1890) and specific plaque hypothesis by Loesche $(1976){ }^{[10]}$. The critical issues can be enlisted as follows:

1. Are a dozen or more microbial species in fact involved in 'a meaningful way' in the etiology of human periodontitis?

Different possibilities like

a) One or two of the species could actually cause the disease. Others are propagators/by standers. Evidence of a 'mixed infection'. The significance of this issue is that the treatment may be different than that for treating multiple bacteria. 
b) Periodontitis as a consequence of sequential infection. Host response to particular species will prevent recurrence of infection by that species. But another species can cause infection.

c) Multiple species of bacteria may share a common characteristic or factor that is the immediate cause of the disease. E.g. - sharing a common virulence factor like LPS.

2. Are periodontal infections a consequence of overgrowth of commensal periodontal micro flora or exogenous infections?

Supporting theory is that transmission may not be a critical issue. In such cases, attention should be focused on determining factors that account for and permit overgrowth to occur at some sites in some individuals but not in others. But there is a contrasting theory that if the bacteria must be acquired for infection to occur, then transmission is a key issue. It needs to be addressed for complete elimination of pathogen. Hence focus needs to be shifted on "infected families" than individuals. ${ }^{[6]}$

3. Question of the relationship between the presence of a "pathogenic" flora and disease status.

While $P$. gingivalis and other putative periodontal pathogens may be found at high frequency in periodontally normal individuals and sites, they may well be a virulent nonpathogenic strains or clonal types. Resolution of this issue is difficult because of the clonicity.

4. Role of environment on bacterial gene expression: major determinant of virulence and needs to be controlled. [11]

\section{c. Issues in pathogenesis}

The line of thinking in 1960's was that periodontitis is an infectious disease. Further, in the decade of the 1970's, researchers focused on the role of the host. The first major milestone was publication by Ivanyi and Lehner (1970) of a paper demonstrating that peripheral blood mononuclear cells from patients with periodontitis were sensitized to antigens of their infecting periodontal bacteria. This observation served as a catalyst for investigators world-wide to focus on the role of host defense mechanisms in the etiology of periodontitis. ${ }^{[12]}$

In the 1980's the possible role and importance of B-cells and the humoral immune response became a focus due to enzyme-linked immunosorbent assay. Enormous advances were made in the 1980's in the understanding of mechanisms of tissue destruction in chronic inflammatory diseases, including periodontitis. Understanding the pathogenesis of disease will make the clinicians' device a custom- made treatment plan for the patients. ${ }^{[11]}$

\section{d. Issues in Diagnosis}

Diagnostic procedures may be used to identify people at risk of dev eloping disease (at risk), detect early stage disease in clinically asymptomatic individuals (screening), classify disease categories (classification), predict likely responders to specific treatments (treatment planning), monitor treatment efficacy and detect disease recurrence (monitoring).

Identification of risk indicators and factors is of enormous importance in diagnosis and treatment planning of periodontitis patients. Critical issue is that there is no evidence-based understanding of association with periodontal disease. Hence there is a need for more longitudinal studies on this front. ${ }^{[13]}$

The quality of the data derived from clinical trials depends on the experience, knowledge and skills of the clinical examiner(s) who are evaluating the patients. There is a lack of training and calibration of clinical examiners for accurate diagnosis. Many of the scoring systems used (such as plaque and gingival indices) are somewhat subjective and there is much scope for interpretation of the scoring criteria by the clinician. Objective scores like probing depths and recession are subject to error by position of probes and reading of scores. Various attempts have been made to conquer these problems such as

- Calibration of examiners: In reference to a fixed scale or a defined set of standards, or even a gold standard

- Gold standard clinician is experienced, knowledgeable and they have low variability in their repeated measures. But cannot be always precise and accurate

- Concept of "Examiner Alignment and Assessment" - Training protocol given by Hefti and Preshaw. ${ }^{[16]}$

Diagnosis of disease activity is the next issue in hand. Concept in 1960's-1970's was that once pocket is formed, disease progresses linearly and continuously to cause eventual loss of tooth. In the 1980's, it was said that disease progression found to be rather episodic, site specific, and infrequent. Observations of studies are as follows:

1. In early to moderate disease, $3-10 \%$ sites worsened to a more debilitating form

2. Most deteriorating sites were seen in very few patients as compared to the milder form of disease.

Conventional diagnostic aids were used like pocket depth, attachment level, bleeding, radiographic manifestations of alveolar bone loss. Inability to make the distinction between diseased/ disease active and 
healthy/ disease in-active pockets is the central critical issue in periodontitis. Hence the need large research effort aimed at development of diagnostic methods capable of detecting disease-active sites. Advanced diagnostic aids in microbial diagnosis can be utilized which have shown promising results.

\subsection{Non-surgical therapy}

\section{Issues In Therapy}

Thoroughness of debridement of the tooth roots of microbial deposits was a more important determinant of the final therapeutic outcome than the treatment modality used. Hence arose the concept of scaling and root planning. An era of adjunctive chemotherapy using systemically administered antibiotics slowly gained popularity. Different obstacles faced by the researchers were the choice of drugs to be used amongst the wide array of available products. Substantivity of these drugs became a debatable issue even after using it for periodontal therapy. Lindhe et al. (1984) claimed that surgical therapy formed an essential part of the treatment plan, thus making the non-surgical methods take a back seat. ${ }^{[14]}$

\subsection{Regenerative therapy}

Outcomes of various grafting procedures in Guided Tissue regeneration are unpredictable. There is a need of extensive basic and clinical research aimed at improving the success rate. Lack of understanding of why some patients fail to respond favorably to any form of periodontal therapy is seen. Goal of research should be to understand the complex host-defense response against this multifactorial disease.

1. Exploring area of immunization.

2. Use of growth factors

3. Gene-delivery approaches

A critical issue that the clinicians face in the regenerative surgical approach is whether periodontitis patients produce antibodies to their infecting bacteria and if not, why? If so, are they protective and if not, why? More than half individuals cannot produce antibodies, if produced, they are non-functional and cannot opsonize or phagocytose. Different solutions have been proposed to overcome this issue. SRP causes bacteraemia and hence can induce active immunization in form of vaccines. Antibodies could be more efficient than those produced spontaneously by local infection.

\subsection{Tissue Engineering}

Desired outcome after periodontal therapy is reconstruction of lost tissues with fully functional apparatus. Issue is to ensure that the correct cell type develops in the correct location with appropriate anatomical orientation, correct physiological and homeostatic functions. One of the promising areas for development is stem cells. Stem cells are induced pluripotent stem cells generated from somatic cells through the forced expression of key transcription factors

\subsection{Recession Coverage}

First of the two basic dilemma is whether to use a graft or not. There are a variety of grafts available to be used in mucogingival surgeries, which can be broadly classified as autogenous and allogenous. Each of them have their own advantages and disadvantages, hence the choice of the material becomes a critical issue. It becomes a necessity to weigh the pros and cons of the material before using it in a clinical set-up. Grafts from donor autogenous sites require adequate amount of tissue available, more surgical time, increased postoperative pain and discomfort, and donor-site morbidity. Wang et. al advocates use of collagen membranes since their advantages are unlimited availability of the grafting product, uniform thickness of the membrane. Hence they qualify as a reliable alternative.

Second critical issue in recession coverage is the choice of the surgical technique to be employed. Many surgical techniques are available; choice can be made depending on the objectives of treatment. Cortellini and Pini Prato have proposed a decision making tree which might help the clinician to decide the approach for recession coverage. (Fig. 1) ${ }^{[15]}$
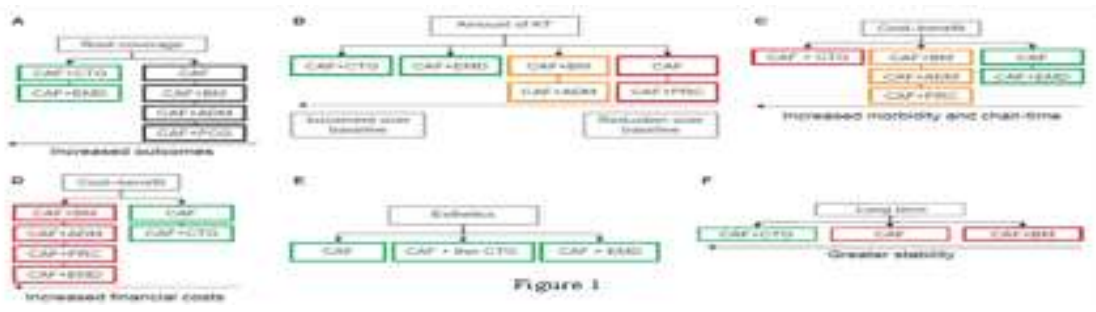


\subsection{Implants or Teeth?}

Factors to be considered while placing implants for replacement of missing teeth include quality of life, esthetics, cost and attitude of patients (as well as many dentists). There is a common thinking that 'once an implant is placed, all worries about oral health are gone.' But an increasing incidence of peri-implantitis is often experienced by the clinicians. Varying concepts of pathogenesis of peri-implantitis are proposed. There are no longitudinal studies on implant systems as conventional periodontal treatments. Hence it is the duty of the practitioner to advise the following

1. Maintain periodontally compromised natural teeth as opposed to maintaining of implants and the risk for peri-implantitis.

2. Clinical risk-assessment procedure

3. When placing an implant, need to assess risk of failure at Implant level, At the site level and At the patient level

4. Clear clinical protocol for the maintenance care

\section{Issues in statistics}

Statistics is a completely different world, with its own language, rules and regulations. Dental researchers and statisticians generally existed in isolation, perhaps interfacing only occasionally, usually with limited understanding on both sides. Biostatisticians are breaking the barriers between clinicians, researchers and statisticians. They have knowledge of, and interest in, dental and periodontal research. Statistics is not just a testing process, it is a thinking process.

\section{Ethical Issues}

In clinical trials, there are failures to obtain Institutional Review Board approval. Plagiarism amongst the members involved in the study or outside is rampant. Lack of co-ordination between Animal ethical review board and clinical researchers, non-availability of animals and strict rules and regulations due to advent of animal rights are seen. Ill-treatment of animals leads to no definite assessment of intervention. Non publishing of potentially useful data is also one of the critical issues.

The available research data and future data should be subjected to intense scrutiny to separate sham research from original. Meticulous evaluation of dissertations should be done in Dental institutions. Studies have to be subjected to meta-analysis and published in accessible reputed journals. Results can be deciphered by proper orientation of students and staff through basic training and screening of research for authenticity and appropriate utilization of funds. ${ }^{[16]}$

\section{Conclusion}

Evidence-based dentistry: Evaluation of the scientific literature, interpretation of this information can be applied in the context of the particular clinical situation which improves the quality of patient care. Clinicians need to develop the art of understanding evidence, strength and weaknesses of studies. Systematic approach towards reviewing literature should be undertaken. One should be up to date with recent advances.

Scientific enquiry is one of the most challenging enterprises of mankind. A definite collaborative strategy could bring about radical changes in the public perception and understanding of periodontal research. Periodontal research in India has to create its own identity in the global scenario. The ultimate aim of the research should be to benefit the so called 'common man'.

\section{References}

[1]. Gies WJ. Dental education in the United States and Canada. New York: Carnegie Foundation for the Advancement of Teaching, 1926

[2]. Kothari CR. Research methodology and techniques. 2nd ed. Research methodology: An introduction, Chapter 1, 1-29. Wishwa prakashan. New Delhi.

[3]. Pihlstrom BL, Curran AE, Voelker HT, Kingman A. Randomized controlled trials: What are they and who needs them? Periodontol 2000 2012;59:14-31.

[4]. Russell AL. The periodontal index. J Periodontol 196738(Part II):585-591.

[5]. Ramfjord SP. Periodontal disease index (PDI). J Periodontol 1967 38(Part II):602-610.

[6]. Page RC. Critical issues in periodontal research. J Dent Res 1995;74:1118-28

[7]. Baelum V, Fejerskov 0, Karring T. Oral hygiene, gingivitis, and periodontal breakdown in adult Tanzanians. I Periodont Res 1986 21:221-232.

[8]. Reddy J, Africa CW, Parker JR. Darkfield microscopy of subgingival plaque of an urban black population with poor oral hygiene. J Clin Periodontol 1986 13:578-582.

[9]. Loe H, Theilade E, Jensen SB. Experimental gingivitis in man. J Periodontol 1965;36:177-87.

[10]. Loesche WJ (1976). Chemotherapy of dental plaque infection. Oral Sci Rev 9:65-107.

[11]. Page RC. Milestones in periodontal research and the remaining critical issues. J Periodontal Res 1999;34:331-9.

[12]. Ivanyi L, Lehner T. Stimulation of lymphocyte transformation by bacterial antigens in patients with periodontal disease. Arch Oral Biol 1970; 15:1089-1096.

[13]. Mombelli A. Critical issues in periodontal diagnosis. Periodontol 2000 2005;39:9-12. 
[14]. Lindhe J, Westfelt E, Nyman S, Socransky SS, Haffajee AD. Long-term effect of surgical/non-surgical treatment of periodontal disease. J Clin Periodontol 1984 11:448-458.

[15]. Pini-Prato, G. P., Clauser, C. \& Cortellini, P. Periodontal plastic and mucogingival surgery. J Periodontol 2000. 1995; 9, 90-105

[16]. Preshaw PM. Critical issues in clinical periodontal research. Periodontol 2000 2012;59:7-13. 